\title{
Comparison of Optical Single Sideband Techniques for THz-over-fiber Systems
}

\author{
Luis Gonzalez-Guerrero, Haymen Shams, Member, IEEE, Irshaad Fatadin, Senior Member, IEEE, \\ Martyn J. Fice, Member, IEEE, Mira Naftaly, Alwyn J. Seeds, Fellow, IEEE, and Cyril C. Renaud, \\ Senior Member, IEEE
}

\begin{abstract}
The use of single sideband (SSB) signals and envelope detection is a promising approach to enable the use of economic free-running lasers in photonic THz communications. To combat the signal-signal beat interference (SSBI) associated with envelope detection, broad guard bands (GBs) may be used given the large unregulated spectrum available at $\mathrm{THz}$ frequencies $(100 \mathrm{GHz}-10$ THz). In this scenario, the conventional way of generating SSB signals through a digital SSB filter (here referred to as the CSSB scheme) would require quite high analog digital-to-analog converter (DAC) bandwidths. Digital virtual SSB (DVSSB) and analog virtual SSB (AVSSB) have been proposed in directdetection optical systems for relaxing the DAC bandwidth requirements. In this paper, we compare the three techniques through simulations and implement them, for the first time, in a THz-over-fiber (ToF) system operating at $250 \mathrm{GHz}$. For the transmission experiments we employ 5 GBd 16-QAM signals with three different GBs (5.5 GHz, 4.75 GHz and 3.5 GHz). The simulations show that the best performance is obtained with the AVSSB technique, while the worst is obtained with the DVSSB scheme, where the quality of the generated sideband degrades with carrier-to-sideband power ratio. In the experimental transmissions, where receiver noise was the main source of noise, similar behavior was found between the three techniques. At the 3.5 GHz GB, however, the DVSSB exhibited a penalty of $1 \mathrm{~dB}$ with respect to the other two. This is likely to be due to nonlinear distortions caused by the increase in the virtual tone power.
\end{abstract}

Index Terms-Broadband communication, digital signal processing, envelope detectors, microwave photonics, millimeter wave communication, optical mixing, photonic integrated circuits, semiconductor lasers.

\section{INTRODUCTION}

$\mathrm{F}$ OR $5 \mathrm{G}$ mobile systems, data rates of up to $100 \mathrm{Gbit} / \mathrm{s}$ will be required in the backhaul links of both wireless and optical access networks [1]. Although optical fiber is likely to be the preferred option for this type of link due to its large bandwidth, its deployment will not always be economical, as

"This work was supported by the Engineering and Physical Sciences Research Council through the Coherent Terahertz Systems (COTS) (EP/J017671/1) and COALESCE (EP/P003990/1) grants, and by the European Commission through the European project iPHOS (grant agreement no: 257539). This project has also received funding from the European Union's Horizon 2020 research and innovation programme under grant agreement 761579 (TERAPOD). The work was also supported by the project EMPIR 14IND13 PhotInd at NPL. This project has received funding from the EMPIR programme co-financed by the Participating States and from the European Union's Horizon 2020 research and innovation programme." for example in crowded urban areas or difficult-to-access terrains. In this scenario, a high-speed wireless link at $\mathrm{THz}$ frequencies $(100 \mathrm{GHz}-10 \mathrm{THz})$ can be advantageous. Compared to mm-wave systems, the large bandwidth available at $\mathrm{THz}$ frequencies would allow the use of low-order modulation formats, which require less power at the transmitter, with important advantages in energy efficiency and health and safety issues. On the other hand, unlike free-space optical (FSO) communications, $\mathrm{THz}$ links would ensure operability under adverse atmospheric conditions such as fog, or in the presence of airborne particles, such as smoke or dust.

At the same time, trends indicate that optical and wireless networks will converge to a highly-integrated network [2]. As such, the prospect of seamless integration with fiber networks will be a very important requirement for wireless stations in $5 \mathrm{G}$ networks. In this scenario, radio-over-fiber (RoF) techniques based on the heterodyne of two lasers in a photodiode offer a substantial advantage over fully electronic $\mathrm{THz}$ emitters in terms of system architecture simplicity and cost [3],[4]. A drawback of photonic mixing is that the resulting wireless signal exhibits a phase noise equal to the sum of the two laser linewidths. To solve this problem there are two approaches: (a) using phase noise-correlated optical tones for data carrier and pilot tone or (b) using amplitude modulation \& envelope detection [5]-[7].

Unlike for lower radio frequencies (RFs), techniques based on the double sideband-suppressed carrier (DSB-SC) scheme cannot be used for $\mathrm{THz}$ generation given the frequency limitation of available sources and optical modulators. Hence, for the first approach, solutions normally rely on the generation of an optical frequency comb and the subsequent filtering of two comb lines [8]. This technique increases considerably the complexity of the transmitter and requires the use of a compensating fiber to account for the optical length mismatch that arises when inserting an optical modulator in one of the optical paths [9]. The second technique, on the other hand,

L. Gonzalez-Guerrero, H. Shams, M. J. Fice, A. J. Seeds and C. C. Renaud are with the Department of Electronic and Electrical Engineering, University College London, Torrington Place, London, WC1E 7JE, England (e-mail: uceelgo@ucl.ac.uk; h.shams@ucl.ac.uk;_m.fice@ucl.ac.uk; a.seeds@ucl.ac.uk; c.renaud@ucl.ac.uk).

I. Fatadin and M. Naftaly are with the National Physical Laboratory, Teddington, TW11 0LW, U.K. (email: irshaad.fatadin@npl.co.uk; mira.naftaly@npl.co.uk). 
enables the use of free-running lasers, which remain attractive to internet providers because of their simplicity, low cost and tuneability capabilities [10]. To increase the spectral efficiency of this scheme, data can be modulated onto a RF subcarrier, which enables the use of quadrature amplitude modulation (QAM). A further increase in optical spectral efficiency, together with an improved tolerance to dispersion degradation, can be achieved by suppressing one of the redundant sidebands (i.e. by using the single sideband, SSB, modulation format) [11]. In a recent paper, we demonstrated the phase noise insensitivity of this scheme in a $\mathrm{THz}$ system using two broadlinewidth lasers [12]. Apart from enabling the use of low cost lasers, this scheme also relaxes the complexity of the digital signal processing (DSP) operations aimed at recovering the phase of the transmitted signal. Furthermore, a receiver based on envelope detection, unlike those based on RF mixing, does not require a local oscillator (LO), which further simplifies the system architecture.

One of the issues with SSB envelope-detected signals is the signal-signal beat interference (SSBI) that results from the overlap of the direct detection (DD) terms and the signal. There are two possible ways to mitigate the SSBI: (a) using SSBIcancelation DSP algorithms at the receiver and (b) allocating a guard band (GB) between carrier and sideband. While the Kramers-Kronig receiver is a very promising solution for DD optical systems [13], the increased DSP associated with this technique can be a critical factor for wireless receivers, which have more stringent power- and cost-requirements. Moreover, at $\mathrm{THz}$ frequencies, given the large unregulated spectrum (up to $70 \mathrm{GHz}$ available in the window centered at $287 \mathrm{GHz}$ [14]), the use of wide GBs may be a reasonable choice. However, considering the large data rates envisaged from $\mathrm{THz}$ communications, this approach can place stringent requirements on the analog bandwidth of the digital-to-analog converter (DAC) used at the transmitter. In [7], the only experiment among those listed before performed at a carrier frequency above $100 \mathrm{GHz}$, the bandwidth of the GB was set equal to that of the baseband signal to entirely cancel the SSBI. Under these circumstances a DAC with an analog bandwidth of at least $20 \mathrm{GHz}$ was required to perform the transmission.

If GBs comparable to the signal bandwidth are to be a feasible approach in $\mathrm{THz} \mathrm{SSB}$ systems, it would be advantageous, thus, to find SSB generation techniques that relax the DAC bandwidth requirements. To do so, one can turn to DD optical networks, where two types of techniques have been proposed for such a purpose: the digital virtual SSB (DVSSB) [15] and the analog virtual SSB (AVSSB) [16]. In this paper, we demonstrate, for the first time, these two techniques in a THz-over-fiber (ToF) system working at $250 \mathrm{GHz}$. Moreover, we compare their performance to the conventional way of generating SSB signals through the Hilbert transform (here referred to as conventional SSB, CSSB). This is to the best of the authors' knowledge the first joined comparison of the three techniques.

The rest of the paper is organized as follows. In section II, each of the SSB signal generation techniques, as well as the receiver DSP and the complete experimental arrangement for
$\mathrm{THz}$ transmission are described. In section III, the quality of the optical signal generated by each technique is studied through simulations. Then all techniques are experimentally demonstrated and their BER performance is analyzed and compared for different GBs. Finally, in section IV, we conclude the paper by highlighting the main features and problems of each technique.

\section{EXPERIMENTAL PROCEDURE}

\section{A. Transmitter}

In this subsection, we review the three schemes employed to generate the THz SSB signal, namely: CSSB, DVSSB and AVSSB. We highlight the bandwidth required by each technique, their operation mechanism, practical implementation issues, and how the carrier-to-sideband power ratio (CSPR) can be adjusted in each of them. SSB signals with low CSPR suffer from high SSBI, while high CSPR leads to reduced signal-to-noise ratio (SNR) [15]. Thus, it is important to ensure the system always operates at the optimum CSPR value. For each technique, three different GBs were tested: $5.5 \mathrm{GHz}, 4.75 \mathrm{GHz}$, and $3.5 \mathrm{GHz}$. The first one was chosen to have the same bandwidth as the baseband signal (a 5 GBd 16QAM signal with a root raised cosine filter roll-off factor of 0.1 ) so that no SSBI was present upon detection. The $4.75 \mathrm{GHz}$ and $3.5 \mathrm{GHz}$ GBs were used to study the ability of each technique to tune the CSPR to combat the SSBI (lower GBs were not accessible due to practical limitations as will be seen next).

1) Conventional SSB (CSSB) implementation

The CSSB transmitter is shown in Fig.1. The baseband signal is up-converted to a subcarrier frequency $f_{S C \text {-CSSB }}$, which is varied according to the desired GB. The up-converted signal is then split into two arms with one of them passing through a sideband filter based on the Hilbert transform. By properly biasing an optical $I Q$ modulator, one of the sidebands can be removed while keeping the optical carrier, which is transmitted along with the signal. In this case, the CSPR of the signal is adjusted by changing the biasing points of the $I Q$ modulator. The total DAC bandwidth required by this technique is $\mathrm{BW}+$ $\mathrm{GB}$, where $\mathrm{BW}$ is the bandwidth of the baseband signal. For the 5.5 GHz GB, the modulator was biased close to the null point to achieve a low CSPR. For the $4.75 \mathrm{GHz}$ and $3.5 \mathrm{GHz} \mathrm{GBs}$, on the other hand, the biasing points were progressively tuned towards the quadrature point to increase the CSPR. 

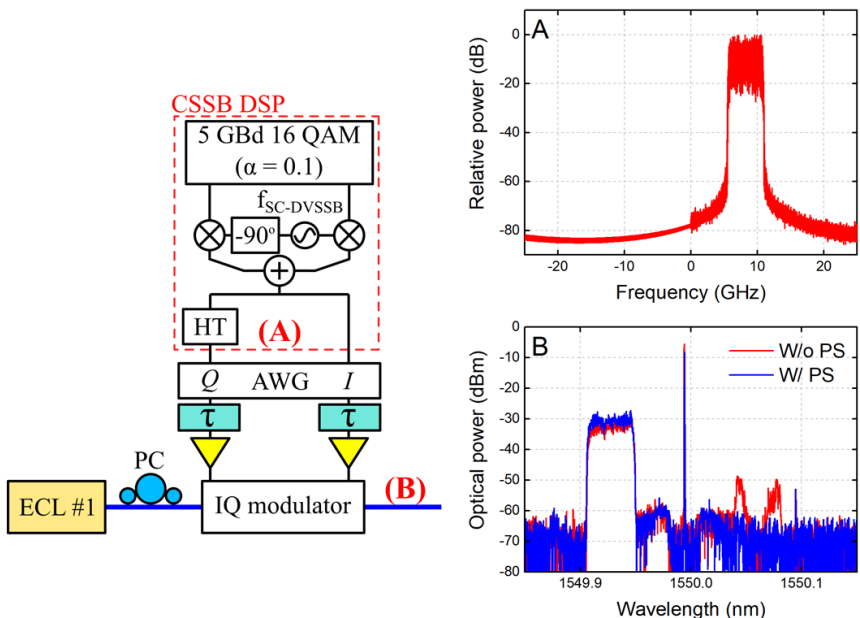

Fig. 1. Optical CSSB transmitter design. Insets: (A) complex spectrum of the digital signal for $f_{\mathrm{SC}-\mathrm{CSSB}}=8.25 \mathrm{GHz}$, and (B) spectrum of the resultant optical signal (blue) with phase shifters before the electrical amplifiers and (red) without them (resolution bandwidth of $10 \mathrm{MHz}$ ). ECL: external cavity laser, PC: polarization controller, AWG: arbitrary waveform generator, PS: phase shifters, and $f_{\mathrm{SC}-\mathrm{CSSB}}$ : subcarrier frequency. $f_{\mathrm{SC}-\mathrm{CSSB}}=\mathrm{GB}+S_{R}(1+\alpha) / 2$, where GB is the guard band, $S_{R}$ is the symbol rate and $\alpha$ is the roll-off factor of the root raised cosine filter.

The complex spectrum generated offline for the $5 \mathrm{GBd} 16$ QAM signal at $f_{\text {SC-CSSB }}=8.25 \mathrm{GHz}$ is shown in Fig. 1 inset (A) with the resultant optical spectrum for this signal shown in Fig. 1 inset (B). When the optical SSB signal is generated with this technique, the optical sideband suppression ratio (OSSR) is approximately equal to the extinction ratio (ER) of the $I Q$ modulator [17], provided the signals on both arms are amplitude-, phase- and time-matched (i.e. there is no $I Q$ imbalance). In our system, there was a time delay between the $I$ - and $Q$-channels. To correct this, two phase shifters (PS) were placed before the RF amplifiers feeding the optical modulator. As can be seen from Fig. 1 inset (B), inserting these PSs was key to achieve the maximum OSSR permitted by our modulator, whose ER was measured to be $30 \mathrm{~dB}$.

2) Digital virtual SSB (DVSSB) implementation

In the DVSSB technique, the tone and the baseband signal are digitally multiplexed (see Fig. 2) and sent to an $I Q$ modulator, in which the $I$ - and $Q$-components must be biased at the null point to suppress the optical carrier [15]. By properly setting the digital tone and subcarrier frequencies $\left(f_{T-D V S S B}\right.$ and $f_{S C-D V S S B}$, respectively), the maximum frequency of the digital signal can be reduced by a factor of 2 compared with that of CSSB (i.e. the required DAC bandwidth is now $\left.1 \frac{1}{2}[\mathrm{BW}+\mathrm{GB}]\right)$. The CSPR is set digitally by varying the amplitude of the digital tone (for a normalized power of the complex baseband signal, $A=$ $\left[10^{(\mathrm{CSPR} / 10)}\right]^{-1 / 2}$, where $A$ is the amplitude of the digital tone). The digital and optical spectra for the $5.5 \mathrm{GHz}$-GB signal are shown in Fig. 2 insets (A) and (B) respectively. As can be seen from the blue trace in Fig. 2 inset (B), the OSSR achieved using this technique is also around $30 \mathrm{~dB}$. The peaks in Fig. 1 inset (B) at $30 \mathrm{~dB}$ below the virtual tone are spurious peaks generated in the AWG.
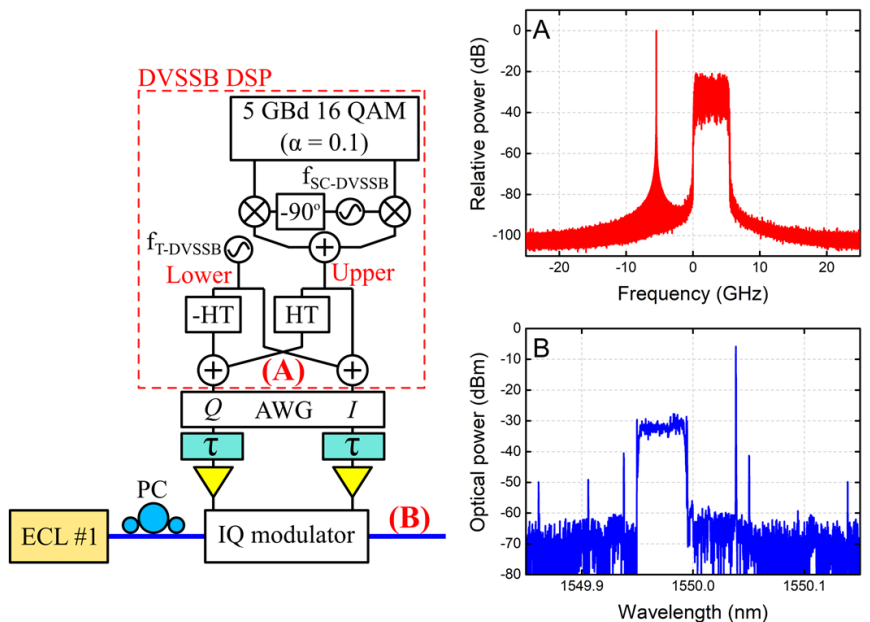

Fig. 2. Optical DVSSB transmitter design. Inset (A) complex spectrum of the offline digital signal for $f_{S C-D V S S B}=2.75 \mathrm{GHz}$ and $f_{T-D V S S B}=-5.5 \mathrm{GHz}$ and (B) spectrum of the resultant optical signal (resolution bandwidth of $10 \mathrm{MHz}$ ). $f_{T-D V S S B}=1 / 2\left[G B+S_{R}(1+\alpha)\right]$ and $f_{S C-D V S S B}=G B / 2$, where $G B$ is the guard band, $S_{R}$ is the symbol rate and $\alpha$ is the roll-off factor of the root raised cosine filter.

\section{3) Analog virtual SSB (AVSSB) implementation}

In the AVSSB scheme, the AWG is used only to generate the baseband signal, which is then multiplexed with an RF tone in the analog domain [16], as shown in Fig. 3. The frequency of this tone $\left(f_{T-A V S S B}\right)$ is equal to the subcarrier frequency of the CSSB technique $\left(f_{S C \text {-CSSB }}\right)$. Since the DAC only generates the baseband signal, the required bandwidth is $\mathrm{BW} / 2$, which compared to that of the DVSSB is a factor of two lower when the GB and BW are the same. As in the DVSSB technique, the $I Q$ modulator bias points are also set at the null point. Ideally, the CSPR is tuned by adjusting only the power from the RF oscillator (but CSPR tuning can also be achieved by decreasing the AWG output power).
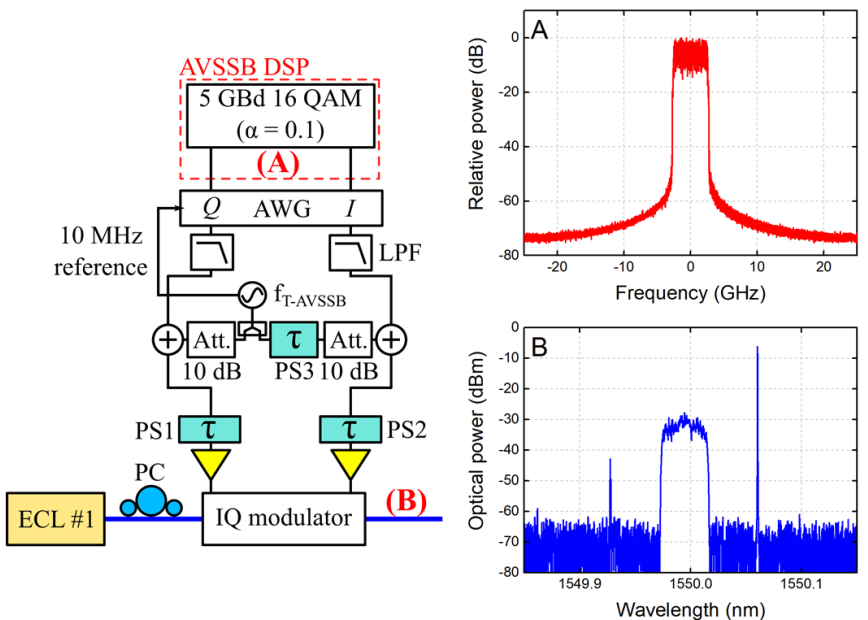

Fig. 3. Optical AVSSB transmitter design. Inset (A) complex spectrum of the offline digital signal, and (B) the resultant optical spectrum for $f_{T-A V S S B}=8.25$ $\mathrm{GHz}$ (resolution bandwidth of $10 \mathrm{MHz}$ ). $f_{T-A V S S B}=G B+S_{R}(1+\alpha) / 2$, where GB is the GB, $S_{R}$ is the symbol rate and $\alpha$ is the roll-off factor of the root raised cosine filter.

The RF oscillator $10 \mathrm{MHz}$ reference output signal was used as the clock signal for the AWG to ensure a good phase relationship between the data subcarrier and analog tone. The phase shifter after the RF oscillator (PS3 in Fig. 3) was adjusted 
to provide a $90^{\circ}$ phase shift to the RF tone. As can be seen, an OSSR between the desired and image optical tones of around $35 \mathrm{~dB}$ was achieved. To achieve a good suppression, both branches of the analog circuit must have the same amplitude and phase response (as no data goes through, time-matching is not as important provided the RF oscillator has sufficiently low phase noise). To prevent the crosstalk between the $I$ - and $Q$ channels, the two branches must also be properly isolated. In our case, using two $10 \mathrm{~dB}$ attenuators was found to be enough to suppress the crosstalk.

Low pass filters (LPF) were used to prevent excessive RF power from going into the AWG output ports. The bandwidth of these LPFs set the minimum value of guard-band allowed by this technique, which is the reason why the $3.5 \mathrm{GHz} \mathrm{GB}$ was chosen as the lower bound in the comparison experiments. The digital and optical spectra generated with this technique for $f_{T-}$ ${ }_{A V S S B}=8.25 \mathrm{GHz}$, are shown in Fig. 3 insets (A) and (B) respectively.

\section{B. Receiver DSP}

The DSP blocks used in the digital receiver are shown in Fig. 5. As mentioned in the introduction, one of the advantages of DD systems is the simplicity of the DSP, since no complex carrier frequency and phase retrieval techniques are needed. In our case, after envelope detection the signal was resampled to 6 samples $(\mathrm{Sa}) /$ symbol and fed into a digital $I Q$ demodulator. After down-conversion, a coherent DSP routine was carried out consisting of: matched filtering, resampling \& normalization, phase recovery and equalization. The equalizer was initialized using the radius directed algorithm (RDE) and then switched to a decision-directed (DD) mode equalizer. Before switching to the DD equalizer, the Viterbi-Viterbi algorithm was applied for phase corrections. Note that this step was only required to compensate for a constant phase offset and a block averaging filter of up to 600 samples in length could be used to remove the effect of noise. This phase offset is caused by the power ratio between the I- and Q-tones generated in each branch of the optical IQ modulator. Its magnitude can be expressed as $\Delta \varphi=$ $\arctan \left[\mathrm{P}_{Q} / \mathrm{P}_{I}\right]^{1 / 2}$, where $\Delta \varphi$ is the phase offset, and $\mathrm{P}_{Q, I}$ are the power of the I-and Q-optical tones respectively. In a practical system, $\Delta \varphi$ may be removed with a single-phase rotation without the Viterbi-Viterbi algorithm to reduce digital complexity [18]. Here, since we were constantly changing the modulator biasing points to adjust the CSPR, the ViterbiViterbi algorithm was used to avoid measuring $\Delta \varphi$ at each CSPR value. It is important to note that neither a frequency offset algorithm nor differential decoding was required to retrieve the signal. After mapping the symbols to bits, the BER was calculated over 196608 bits (corresponding to a signal of $10 \mu$ s after removing some symbols at the beginning and the end of the sequence) by error counting. The constellation diagram of the received signal (for the AVSSB scheme and the 5.5 GHz GB) after normalization, RDE, Viterbi-Viterbi and DD equalizer are shown in Fig. 5A, 5B, 5C and 5D respectively.

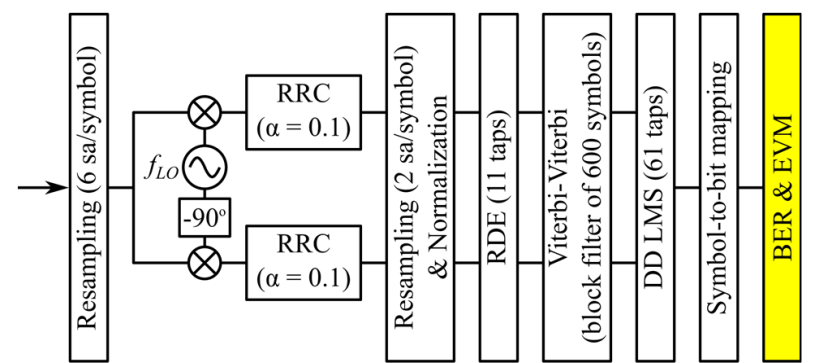

Fig. 4. Receiver DSP block diagram. $f_{L O}$ : LO frequency of the digital demodulator (is the same as $f_{S C-C S S B}$ ). LMS: least mean squares, EVM: error vector magnitude.

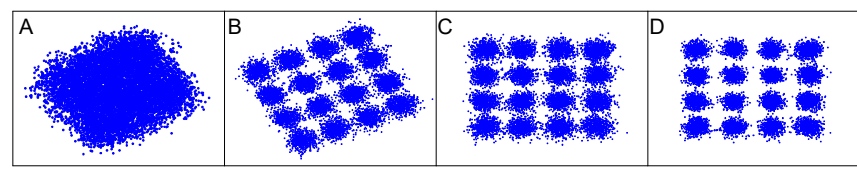

Fig. 5. Received constellation diagram for the AVSSB scheme with the 5.5 GHz GB after (A) normalization, (B) RDE, (C) Viterbi-Viterbi and (D) DD equalizer.

\section{Experimental arrangement}

The complete experimental arrangement used in the transmission experiments is shown in Fig. 6. For digital signal generation, four $2^{11}$ de Bruijn bit sequences were mapped into the $5 \mathrm{GBd}$ 16-QAM symbols using Matlab. The digital waveforms resultant after applying the transmitter DSP described in Section II- $A$, were uploaded to an AWG (Tektronix, AWG70001A) with an analog bandwidth of 12 $\mathrm{GHz}$ and operating at a sampling rate of $50 \mathrm{GSa} / \mathrm{s}$. As shown in Fig. 1-3, the generated waveforms were time-aligned with two phase shifters and electronically amplified (SHF, S807 C) before being fed to the optical modulator. On the transmitter side, an external cavity laser (ECL) with a linewidth of $10 \mathrm{kHz}$ and a wavelength of $1549 \mathrm{~nm}$ was used for data modulation (note that, although narrow-linewidth lasers were used for this comparison, it has been demonstrated that the SSB technique allows the use of broad-linewidth lasers with no associated penalty [12]). The optical signal was then combined with a second ECL with a $10-\mathrm{kHz}$ linewidth and a wavelength of 1551 $\mathrm{nm}$ (frequency difference between the two lasers was 250 $\mathrm{GHz}$ ). After optical amplification and filtering, the two optical tones were fed into an unpackaged uni-travelling carrier photodiode (UTC-PD) by means of a lensed fiber. Horn antennas with a gain of $25 \mathrm{dBi}$ were used for both transmission and reception and placed at $0.1 \mathrm{~m}$ from each other. A pair of lenses were inserted between the two antennas to increase the collimation of the $\mathrm{THz}$ beam. These lenses had a diameter of 50 $\mathrm{mm}$, which gives a theoretical gain of $42 \mathrm{dBi}$ per lens (according to $G=(4 \pi S) / \lambda^{2}$, where $G$ is the gain, $S$ is the area of the lens, and $\lambda$ is the wavelength of the electromagnetic wave [19]). Due to the high directivity of $\mathrm{THz}$ beams, precise and careful alignment between transmitter and receiver was required. Although this alignment was performed manually in this work, techniques combining beamsteering (through scanning mirrors or phased arrays) and active tracking could help achieving a more precise alignment and, hence, a higher power budget in future $\mathrm{THz}$ systems. 


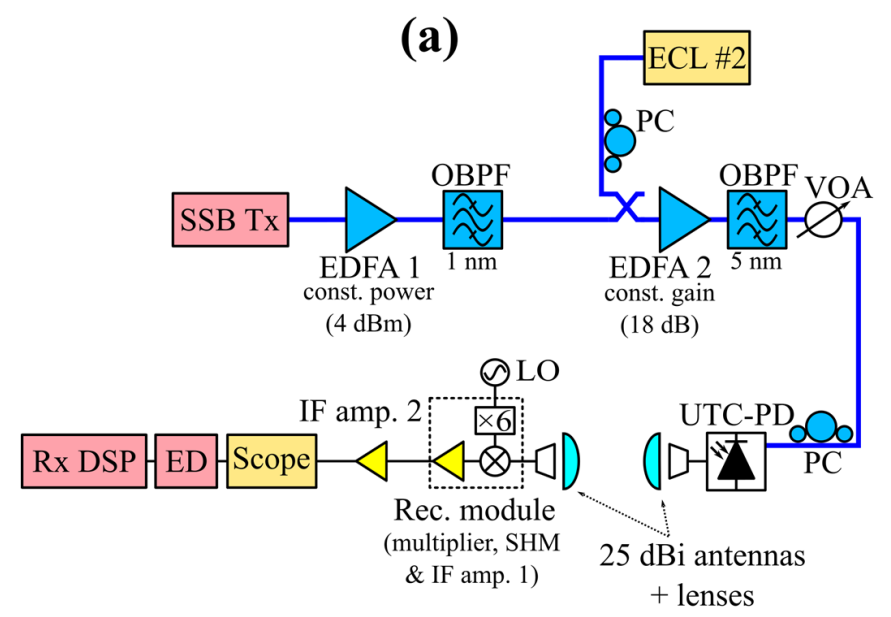

(b)

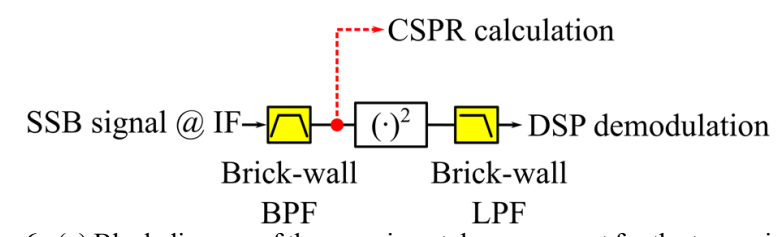

Fig. 6. (a) Block diagram of the experimental arrangement for the transmission of SSB THz signals and (b) block diagram of the envelope detector and point of CSPR calculation. ED: envelope detector, VOA: variable optical attenuator, IF: intermediate frequency, BPF: band pass filter.

On the receiver side, the signal was down-converted to a frequency of around $11 \mathrm{GHz}$ with a module (Virginia Diodes, WR3.4SAX) consisting of a $\times 6$ multiplier, a second harmonic mixer (SHM), and an intermediate frequency (IF) amplifier. After down-conversion, the IF signal was amplified again (Microsemi AML618P3502) and then digitized in an analog-todigital converter (ADC) operating at $80 \mathrm{GSa} / \mathrm{s}$. As shown in Fig. 6, the envelope detector (ED) was implemented here in the digital domain after ADC. This allowed us to accurately calculate the CSPR and the total power of the transmitted SSB signals from the recorded waveforms. The flow chart of envelope detection together with the CSPR measurement point are shown in Fig 6 (b). The ED consists of a brick-wall band pass filter (BPF) to filter out the SSB signal, a squaring operation and a brick-wall LPF to remove the second order terms. All this was carried out at the sample rate of the ADC.

Note that the data rate presented here $(20 \mathrm{Gbit} / \mathrm{s})$ can be scaled in the proposed $\mathrm{THz}$ system through optical multiplexing techniques such as wavelength division multiplexing (WDM) or polarization multiplexing (PM). These techniques have already been applied to THz systems (see for instance [20]) and are equally applicable to our system (the only difference with [20] being that, in our case, each receiver would be formed by an ED instead of a mixer and an LO). Currently, the main factor preventing our system from achieving higher distances and data rates is the available $\mathrm{THz}$ power. This limitation comes from the low power emitted by the UTC-PD (which was measured to be around $-30 \mathrm{dBm}[4]$ ) and the fact that no $\mathrm{THz}$ amplifiers are used either at the receiver or transmitter. Higher power budgets (enabled by THz amplifiers and higher-power UTCs) combined with optical multiplexing techniques are the two elements we believe will allow photonic $\mathrm{THz}$ systems to meet future capacity demands.

\section{SimUlATION AND EXPERIMENTAL RESUltS \& DISCUSSION}

\section{A. Simulation results}

To determine the quality of the signal produced by each technique simulations were carried out in Matlab. Following the experimental configuration shown in Fig. 1-3, the digital waveforms generated for each technique (as explained in Section II- $A$ ) were electronically amplified, fed to an IQ modulator and then optically amplified to a constant output power of $4 \mathrm{dBm}$. For the AVSSB technique, before electronic amplification, the waveform was combined with a tone and passed through a $7 \mathrm{~dB}$ attenuator (emulating the insertion loss of adaptors and cables and the intrinsic $6 \mathrm{~dB}$ loss of the power combiner). The noise of the AWG was modelled as additive white Gaussian noise (AWGN) whose variance was calculated using the parameters provided in the specification sheet and according to

$$
\sigma_{\mathrm{AWG}}^{2}=\left(\frac{\mathrm{V}_{\mathrm{FS}}^{2}}{8}\right)\left(\frac{\mathrm{BW}_{\mathrm{sim}}}{\mathrm{BW}_{\mathrm{SNDR}}}\right) 10^{-(0.602 N+0.176)},
$$

where $\mathrm{V}_{\mathrm{FS}}$ is the full-scale voltage, $\mathrm{BW}_{\text {sim }}$ and $\mathrm{BW}_{\mathrm{SNDR}}$ are the simulation and signal-to-noise-and-distortion ratio (SNDR) measurement bandwidths respectively, and $N$ is the effective number of bits. The noise generated in the electronic circuit was estimated from the noise figure of the transmitter amplifiers ( $5 \mathrm{~dB}$ ) by calculating their equivalent noise temperature. For the AVSSB technique, the noise figure of the attenuator block, which was set as its attenuation value, was also considered in the calculation. The gain of the electronic amplifiers was adjusted to avoid nonlinear distortions in the IQ modulator, which was illuminated with an optical power of $5 \mathrm{dBm}$. As in the experimental transmissions, the output of the modulator was amplified to an optical power of $4 \mathrm{dBm}$ with an EDFA. To compute the amplified spontaneous emission (ASE) noise, a spontaneous factor of 1.5 was used.

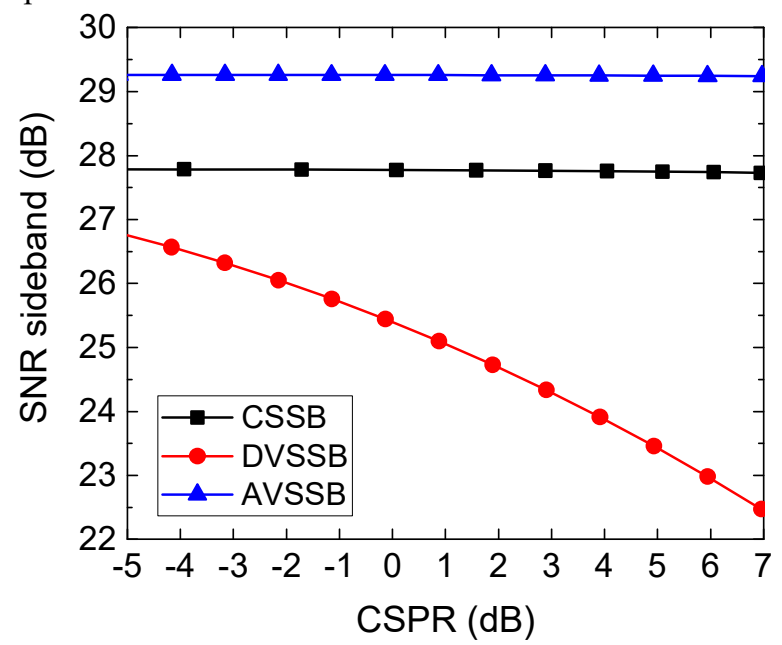

Fig. 7. Optical sideband SNR versus CSPR for each technique.

The SNR of the optical SSB signal was used as the figure of 
merit for the comparison and was evaluated as

$$
\frac{\mathrm{E}\left[|X|^{2}\right]}{\mathrm{E}\left[|Y-X|^{2}\right]},
$$

where $X$ and $Y$ are the symbols generated at the digital transmitter and after optical amplification, respectively, and E[.] denotes statistical expectation. Fig. 7 shows the sideband SNR versus the CSPR for the three techniques. Assuming a linear conversion from the electronic to the optical domain (and neglecting the frequency response of the system), the SNR of the sideband is independent of the GB so only the results for the 5.5 GHz GB are shown. As can be seen from this figure, the SNR of both the AVSSB and CSSB techniques remains constant while that of the DVSSB degrades with CSPR. This is because, unlike in the first two schemes, the electrical power allocated to the sideband in the DVSSB technique decreases as the CSPR is increased. The higher SNR of the AVSSB technique compared to that of the CSSB scheme, accounts for the fact that the average power of the baseband waveform (i.e. the AVSSB technique) was higher than that of the up-converted waveform (CSSB). In the digital waveforms used in the experiment, the difference was $1.8 \mathrm{~dB}$. The lower value found in Fig. 7 between the AVSSB and CSSB curves $(\sim 1.5 \mathrm{~dB})$ comes from the slight SNR degradation that causes the attenuator in the AVSSB signal. Contrary to what could be expected, this attenuation does not degrade much the quality of the signal because of two reasons: (a) the noise from the AWG is much higher than the noise introduced by the attenuator and (b) even attenuated, the electrical noise dominates over ASE noise.

It should be mentioned that, although the SNR and the electrical power of the sideband remain constant in the AVSSB and CSSB techniques, the power of the sideband after optical amplification decreases with higher CSPR in all the techniques. This accounts for the fact that the optical amplifier was operated in a constant output power mode. Noticing this is important because in the transmission experiments, where receiver noise prevailed over transmitter noise, the SNR of the received sideband decreased as the CSPR was increased. Receiver noise was dominated by the large noise figure of the WR3.4SAX module, whose specification value is $24 \mathrm{~dB}$ (corresponding to a noise density of $-150 \mathrm{dBm} / \mathrm{Hz}$ ).

\section{B. Experimental results}

In the previous sub-section, the quality of the generated SSB signal was analyzed through simulations. In this sub-section we compare the quality of the received signals using the experimental arrangement shown in Fig. 6. As noted before, in the $\mathrm{THz}$ transmissions, the main source of noise was receiver noise. As the power of the optical SSB signals was kept constant, similar results can be expected between the three techniques.

In Fig. 8, the obtained BER with each technique is shown as function of the CSPR for the $5.5 \mathrm{GHz}, 4.75 \mathrm{GHz}$ and $3.5 \mathrm{GHz}$ GBs (at an input optical power to the UTC-PD of $12.9 \mathrm{dBm}$ ). As can be seen, in all the cases, the BER reaches a minimum for a certain CSPR value and then it degrades for both lower and higher CSPRs. For the $4.75 \mathrm{GHz}-$ and the $3.5 \mathrm{GHz}-\mathrm{GB}$ transmissions, where the GB was less than the baseband signal bandwidth (BW), the performance degradation at lower CSPR values is caused by the SSBI, as noted in Fig. 8 (B) and 8 (C), respectively. In the case of the transmission with the $5.5 \mathrm{GHz}-$ GB signal, where the GB was equal to the BW (i.e. there was no SSBI), the BER performance deterioration at lower CSPRs is caused by the sideband-noise beating interference [21], as noted in Fig. 8 (A). On the other hand, at higher CSPRs, the system becomes limited by the decrease in SNR that results from the reduction in sideband power (as mentioned before).

In Fig. 9, the optimum CSPR as function of the normalized $\mathrm{GB}(\mathrm{GB} / \mathrm{BW})$ is shown for each technique. As expected the optimum CSPR increases as the GB decreases to tackle the increase of SSBI. As can be seen, the AVSSB technique presents lower values of optimum CSPR for the $5.5 \mathrm{GHz}$ and $4.75 \mathrm{GHz}$ GBs. For a certain GB, a lower optimum value of CSPR means the technique suffers from lower optical SNR (OSNR) [22]. These lower values could be due to the quantized nature of the CSPR measurements or due to a decrease in the transmitted THz power (due to fiber-UTC PD misalignments or polarization mismatches).

In Fig. 10, the BER curves for each GB and technique are plotted as a function of the total received electrical power, as measured from the recorded waveform in the ADC. The CSPR was adjusted to match the optimum one shown in Fig. 9 and then kept fixed over each BER-curve measurement. In Fig. 11, the penalty at the hard-decision forward error correction (HDFEC) limit (BER of $3.8 \cdot 10^{-3}$ ) is shown as function of the normalized GB (GB/BW).

While the absolute performance of the three schemes is very similar for the $4.75 \mathrm{GHz}$ and $5.5 \mathrm{GHz} \mathrm{GBs}$, the penalty substantially increases for the $3.5 \mathrm{GHz}$ GB due to the increased SSBI. In terms of relative performance, one can see that the AVSSB exhibits a slight penalty at the $5.5 \mathrm{GHz}$ and $4.75 \mathrm{GHz}$ GBs but no penalty at the $3.5 \mathrm{GHz}$ GB with respect to the CSSB technique. This penalty (which is less than $0.6 \mathrm{~dB}$ ) could have been caused by a non-optimum CSPR due to the reasons discussed previously. Regarding the DVSSB technique, it can be seen from Fig. 11 that its penalty increases progressively as the GB is reduced, eventually reaching $1 \mathrm{~dB}$ at the $3.5 \mathrm{GHz} \mathrm{GB}$. This could seem to be due to the decrease in SNR with CSPR associated with this technique. However, since transmitter AWGN should not increase with CSPR (i.e. receiver noise still dominates), this is not likely to be the cause. We rather attribute this to nonlinear distortions (in the AWG, electronic amplifier or optical modulator) caused by the increase in the virtual tone power.

\section{SUMMARY \& CONCLUSIONS}

The use of SSB THz signals can allow the use of low-cost free running lasers and enable the use of simplified receivers based on envelope detectors. Considering the large unregulated bandwidth available at $\mathrm{THz}$ frequencies, the use of large GBs may be an acceptable approach to combat the SSBI resulting from envelope detection. In this scenario the bandwidth of 
DACs may become a major limitation to achieve the data rates that are envisaged in future wireless communications. In this paper we have introduced two SSB techniques that relax the bandwidth requirements to the field of $\mathrm{THz}$ communications and compared their performance against the SSB-generation technique that uses the Hilbert transform (in this paper referred to as CSSB). These two techniques, well established in DD optical networks, are digital virtual (DV)-SSB and analog virtual (AV)-SSB.

The quality of the SSB signals produced by each technique is studied through simulations, where we measure the SNR of the optical sideband as a function of the CSPR. We then demonstrate end-to-end system implementations using $5 \mathrm{GBd}$ 16-QAM signals (with a roll off factor of 0.1 and three different GBs: $5.5 \mathrm{GHz}, 4.75 \mathrm{GHz}$, and $3.5 \mathrm{GHz}$ ), which are transmitted in a ToF system operating at $250 \mathrm{GHz}$. The results of the simulations show that, unlike in the CSSB and AVSSB schemes, the quality of the sideband generated with the DVSSB degrades as the CSPR is increased. This is because, in this scheme, the electrical power allocated to the sideband also decreases with CSPR. Between the AVSSB and CSSB techniques, the AVSSB exhibits slightly better SNR as the average power of the baseband waveform is higher than that of the up-converted one. In the transmission experiments, where receiver noise dominates, similar behavior was found between the three techniques. At the $3.5 \mathrm{GHz} \mathrm{GB}$, however, the DVSSB exhibited a penalty of $1 \mathrm{~dB}$ with respect the other two. This is likely to be due to nonlinear distortions caused by the increase in the virtual tone power. As a summary, the main features of each technique, together with the main problem faced by each of them, are summarized in Table I.
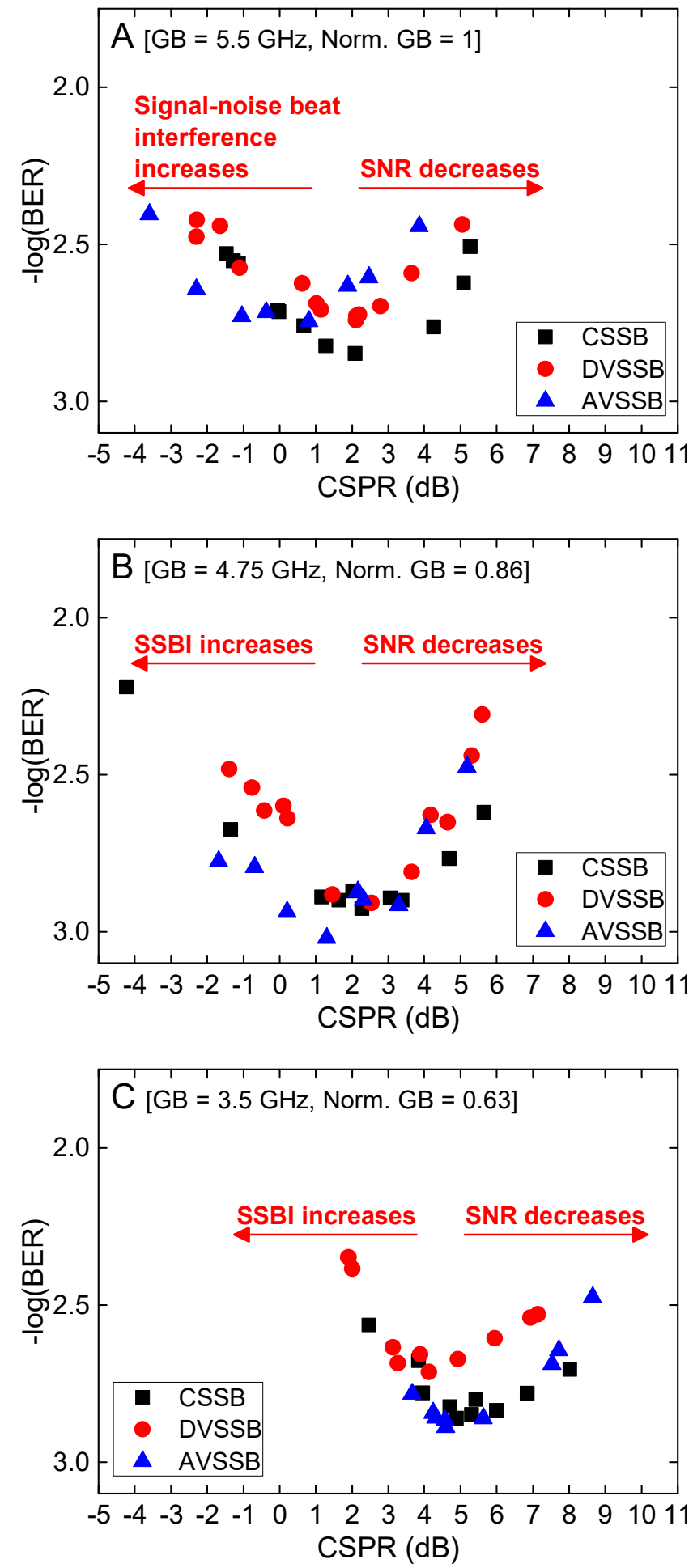

Fig. 8. BER versus CSPR for the (A) $5.5 \mathrm{GHz}$, (B) $4.75 \mathrm{GHz}$, and (C) $3.5 \mathrm{GHz}$ $\mathrm{GBs}$ and at an input optical power to the UTC-PD of $12.9 \mathrm{dBm}$. The normalized $\mathrm{GB}(\mathrm{GB} / \mathrm{BW})$ is also indicated inside the brackets. 


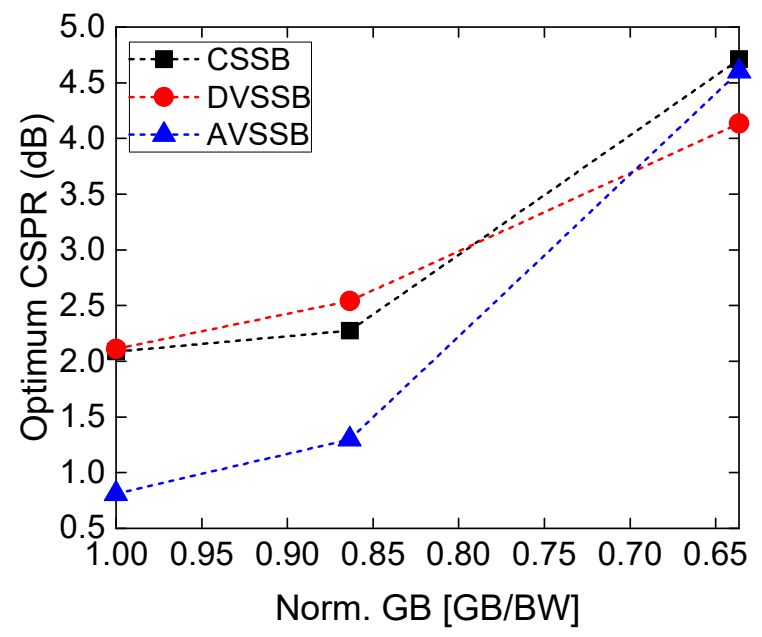

Fig. 9. Optimum CSPR versus normalized GB.

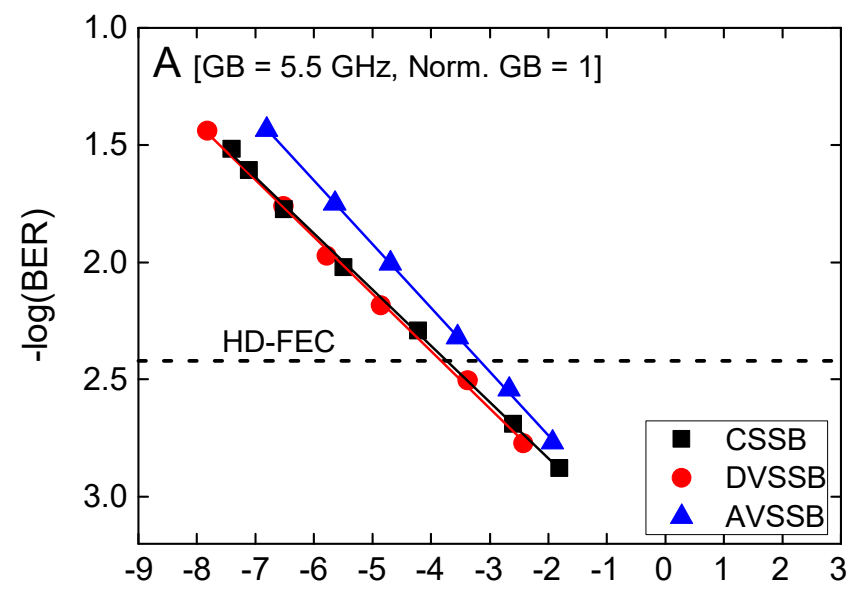

Received electrical power $(\mathrm{dB})$

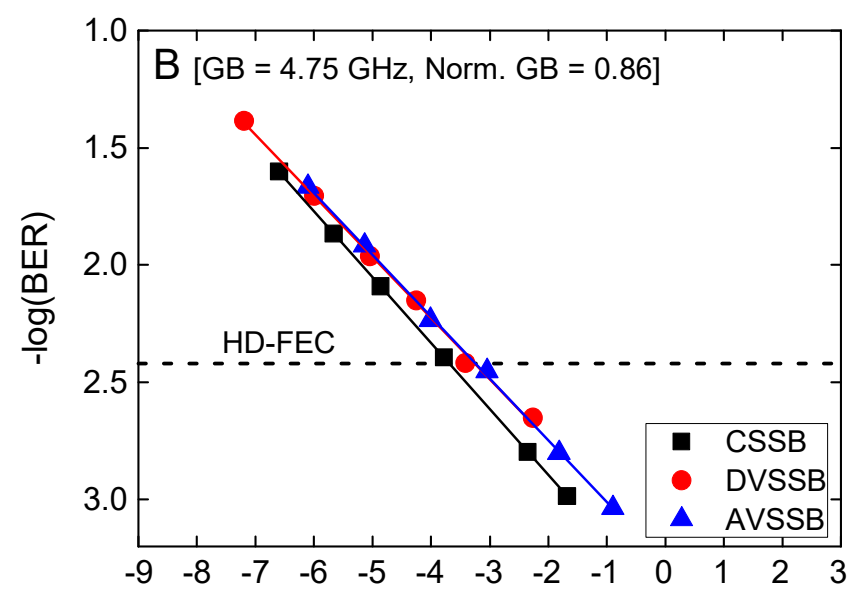

Received electrical power $(\mathrm{dB})$

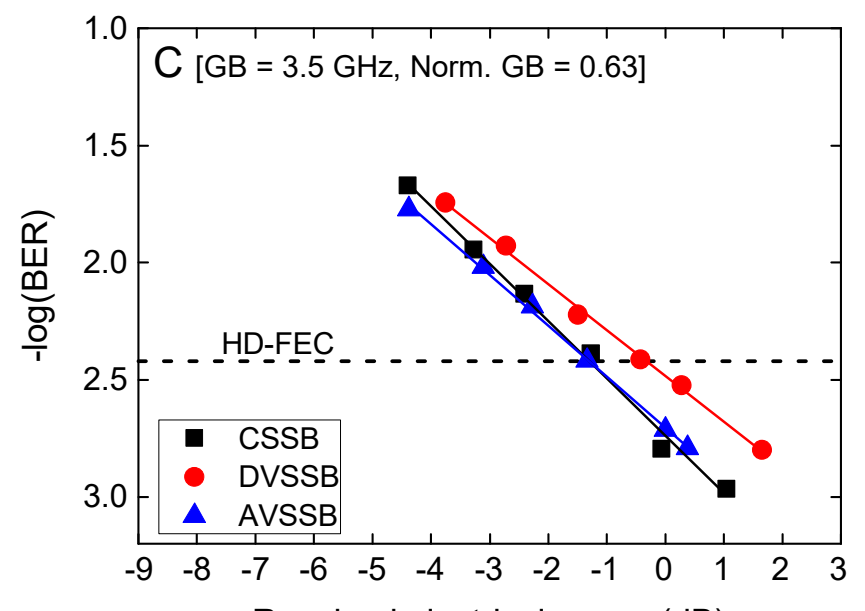

Received electrical power $(\mathrm{dB})$

Fig. 10. BER versus received electrical power for the (A) $5.5 \mathrm{GHz}$, (B) 4.75 $\mathrm{GHz}$, and $(\mathrm{C}) 3.5 \mathrm{GHz} \mathrm{GBs}$. The normalized $\mathrm{GB}(\mathrm{GB} / \mathrm{BW})$ is also indicated inside the brackets. 


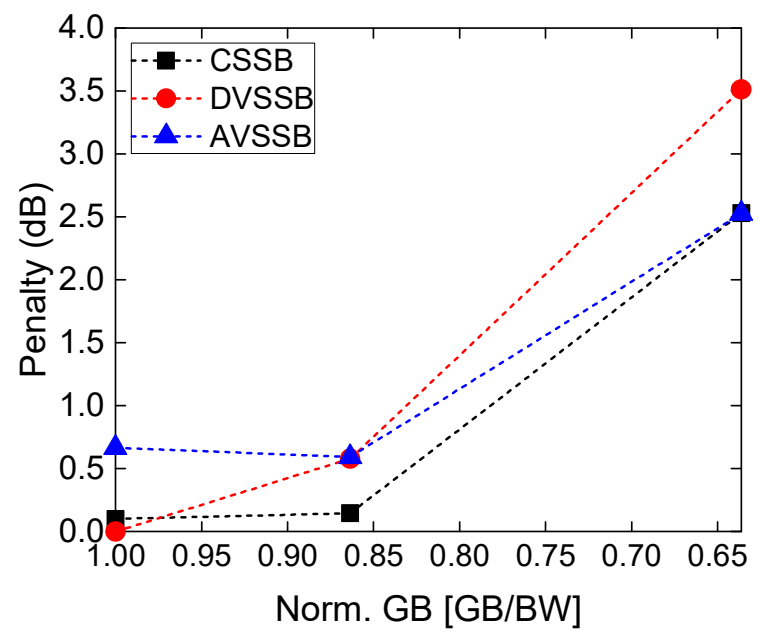

Fig. 11. Penalty (taking the lowest received electrical power yielding a BER below the FEC limit from Fig. 9 as reference) versus normalized GB.

TABLE I

SUMMARY OF SSB TECHNIQUES

\begin{tabular}{cccc}
\hline \hline & CSSB & DVSSB & AVSSB \\
\hline \hline DAC bandwidth & $\begin{array}{c}\text { High } \\
\text { BW }+ \text { GB }\end{array}$ & $\begin{array}{c}\text { Medium } \\
\text { 1/2(BW }+ \text { GB })\end{array}$ & $\begin{array}{c}\text { Low } \\
\text { BW/2 }\end{array}$ \\
$\begin{array}{c}\text { CSPR } \\
\text { tuning }\end{array}$ & $\begin{array}{c}I Q \text {-mod. } \\
\text { biasing points }\end{array}$ & DSP & $\begin{array}{c}\text { RF oscillator } \\
\text { power }\end{array}$ \\
$\begin{array}{c}\text { IQ-mod. } \\
\text { biasing points }\end{array}$ & $\begin{array}{c}\text { According to } \\
\text { desired CSPR }\end{array}$ & Null & Null \\
$\begin{array}{c}\text { DSP } \\
\text { complexity }\end{array}$ & High & High & Low \\
$\begin{array}{c}\text { Analog } \\
\text { complexity }\end{array}$ & Low & Low & High \\
$\begin{array}{c}\text { Main problem } \\
\text { High DAC }\end{array}$ & $\begin{array}{c}\text { Reduction in } \\
\text { sideband SNR } \\
\text { with CSPR }\end{array}$ & $\begin{array}{c}\text { Analog } \\
\text { complexity }\end{array}$ \\
\hline \hline
\end{tabular}

\section{REFERENCES}

S. E. Alavi, M. R. K. Soltanian, I. S. Amiri, M. Khalily, A. S. M. Supa'at, and H. Ahmad, "Towards 5G: A Photonic Based Millimeter Wave Signal Generation for Applying in 5G Access Fronthaul," Sci. Rep., vol. 6, p. 19891, 2016.

[2] W. Ampalavanapillai, Nirmalathas; Prasanna, A. Gamage; Christina, Lim; Dalma, Novak; Rodney, "Digitized Radio Over Fiber Technologies for Converged Optical Wireless Access Network," J. Light. Technol., vol. 28, no. 16, pp. 2366-2375, 2010.

[3] A. J. Seeds, H. Shams, M. J. Fice, and C. C. Renaud, "TeraHertz photonics for wireless communications," J. Light. Technol., vol. 33, no. 3, pp. 579-587, 2015.

[4] H. Shams, M. J. Fice, L. Gonzalez-Guerrero, C. C. Renaud, F. Van Dijk, and A. J. Seeds, "Sub-THz wireless over fiber for frequency band 220-280 GHz," J. Light. Technol., vol. 34, no. 20, pp. 47864793, 2016.

[5] I. Aldaya, G. Campuzano, C. Gosset, and G. Castanon, "Phaseinsensitive RF envelope detection allows optical heterodyning of MHz-linewidth signals," IEEE Photonics Technol. Lett., vol. 25, no. 22, pp. 2193-2196, 2013.

[6] K. Balakier, M. P. Thakur, F. Van Dijk, M. Lamponi, M. Chtioui, Y. Leiba, J. E. Mitchell, A. J. Seeds, and C. C. Renaud, "Demonstration of photonic integrated Rau for millimetre-wave gigabit wireless transmission," in IEEE Topical Meeting on Microwave Photonics (MWP), 2016, vol. 6, pp. 344-347.
[7] M. F. Hermelo, P.-T. (Boris) Shih, M. Steeg, A. Ng'oma, and A. Stöhr, "Spectral efficient 64-QAM-OFDM terahertz communication link," Opt. Express, vol. 25, no. 16, p. 19360, 2017.

H. Shams, T. Shao, M. J. Fice, P. M. Anandarajah, C. C. Renaud, F. Van Dijk, L. P. Barry, and A. J. Seeds, "100 Gb/s multicarrier THz wireless transmission system with high frequency stability based on a gain-switched laser comb source," IEEE Photonics J., vol. 7, no. $3,2015$.

[9] T. Shao, H. Shams, P. M. Anandarajah, M. J. Fice, C. C. Renaud, F. Van Dijk, A. J. Seeds, and L. P. Barry, "Phase Noise Investigation of multicarrier Sub-THz Wireless Transmission System Based on an Injection-Locked Gain-Switched Laser," IEEE Trans. Terahertz Sci. Technol., vol. 5, no. 4, pp. 590-597, 2015.

[10] S. Mandelli, A. Gatto, M. Magarini, P. Boffi, P. Martelli, S Pecorino, and A. Spalvieri, "Phase noise impact on directly detected optical OFDM transmission in uncompensated links," in International Conference on Transparent Optical Networks, 2016, vol. 2016-Augus, no. 2, pp. 5-8.

[11] C. Lim, A. Nirmalathas, M. Bakaul, P. Gamage, K. L. Lee, Y. Yang, D. Novak, and R. Waterhouse, "Fiber-wireless networks and subsystem technologies," J. Light. Technol., vol. 28, no. 4, pp. 390405, 2010.

[12] L. Gonzalez Guerrero, H. Shams, J. Martyn, A. J. Seeds, C. C. Renaud, I. Fatadin, and M. Naftaly, "Experimental Investigation of Phase Noise Tolerance of SSB THz Signals," in IEEE Topical Meeting on Microwave Photonics (MWP), 2017.

[13] Z. Li, M. S. Erkilinc, K. Shi, E. Sillekens, L. Galdino, B. C. Thomsen, P. Bayvel, and R. I. Killey, "SSBI mitigation and the kramers-kronig scheme in single-sideband direct-detection transmission with receiver-based electronic dispersion compensation," J. Light. Technol., vol. 35, no. 10, pp. 1887-1893, 2017.

[14] IEEE 802.15, "TG3d Technical Requirements Document," 2016. [Online]. Available:

http://www.ieee802.org/15/pub/index_TG3d.html.

[15] W.-R. Peng, X. Wu, K.-M. Feng, V. R. Arbab, B. Shamee, J.-Y. Yang, L. C. Christen, A. E. Willner, and S. Chi, "Spectrally efficient direct-detected OFDM transmission employing an iterative estimation and cancellation technique," Opt. Express, vol. 17, no. 11, p. 9099, 2009.

[16] C. Ju, N. Liu, X. Chen, and Z. Zhang, "SSBI Mitigation in A-RFTone-Based VSSB-OFDM System with a Frequency-Domain Volterra Series Equalizer," J. Light. Technol., vol. 33, no. 23, pp. 4997-5006, 2015.

[17] M. S. Erkilinc, M. P. Thakur, S. Pachnicke, H. Griesser, J. Mitchell, B. C. Thomsen, P. Bayvel, and R. I. Killey, "Spectrally efficient WDM nyquist pulse-shaped subcarrier modulation using a dualdrive Mach-Zehnder modulator and direct detection," J. Light. Technol., vol. 34, no. 4, pp. 1158-1165, 2016.

[18] Y. Zhu, M. Jiang, X. Ruan, C. Li, and F. Zhang, " $112 \mathrm{~Gb} / \mathrm{s}$ SingleSideband PAM4 WDM Transmission over 80km SSMF with Kramers-Kronig Receiver," in Optical Fiber Communcation, 2018.

[19] G. Ducournau, P. Szriftgiser, A. Beck, D. Bacquet, F. Pavanello, E. Peytavit, M. Zaknoune, T. Akalin, and J. Lampin, "Ultrawide bandwidth single channel $0.4 \mathrm{THz}$ wireless link combining broadband quasi-optic photomixer and coherent detection," IEEE Trans. Terahertz Sci. Technol., vol. 4, no. 3, pp. 328-337, 2014.

[20] X. Li, J. Yu, K. Wang, M. Kong, W. Zhou, Z. Zhu, C. Wang, and M. Zhao, " $120 \mathrm{~Gb} / \mathrm{s}$ Wireless Terahertz-Wave Signal Delivery by $375 \mathrm{GHz}-500 \mathrm{GHz}$ Multi-Carrier in a 2x2 MIMO System," J. Light. Technol., vol. PP, no. c, p. 1, 2018.

[21] A. J. Lowery, "Amplified-spontaneous noise limit of optical OFDM lightwave systems," Opt. Express, vol. 16, no. 2, p. 860, 2008.

[22] M. S. Erkilinc, Z. Li, S. Pachnicke, H. Griesser, B. C. Thomsen, P. Bayvel, and R. I. Killey, "Spectrally Efficient WDM Nyquist PulseShaped 16-QAM Subcarrier Modulation Transmission with Direct Detection," J. Light. Technol., vol. 33, no. 15, pp. 3147-3155, 2015. 